\title{
Disruption of visual feature binding in working memory
}

\author{
Taiji Ueno • Richard J. Allen • Alan D. Baddeley • \\ Graham J. Hitch • Satoru Saito
}

Published online: 3 November 2010

(C) The Psychonomic Society 2010

\begin{abstract}
In a series of five experiments, we studied the effect of a visual suffix on the retention in short-term visual memory of both individual visual features and objects involving the binding of two features. Experiments 1A, 1B, and 2 involved suffixes consisting of features external to the to-be-remembered set and revealed a modest but equivalent disruption on individual and bound feature conditions. Experiments $3 \mathrm{~A}$ and $3 \mathrm{~B}$ involved suffixes comprising features that could potentially have formed part of the to-be-remembered set (but did not on that trial). Both experiments showed greater disruption of retention for objects comprising bound features than for their individual features. The results are interpreted as differentiating two components of suffix interference, one affecting memory for features and bindings equally, the other affecting memory for bindings. The general component is tentatively identified with the attentional cost of operating a filter to prevent the suffix from entering visual working memory,
\end{abstract}

T. Ueno $(\bowtie)$

Neuroscience and Aphasia Research Unit (NARU),

School of Psychological Sciences, University of Manchester,

Room T4, 3rd Floor, Zochonis Building, Brunswick Street,

Manchester M13 9PL, United Kingdom

e-mail: taiji.ueno@postgrad.manchester.ac.uk

R. J. Allen

University of Leeds,

Leeds, UK

A. D. Baddeley · G. J. Hitch

University of York,

York, UK

T. Ueno $\cdot$ S. Saito

Kyoto University,

Kyoto, Japan whereas the specific component is attributed to the particular fragility of bound representations when the filter fails.

Keywords Working memory · Feature binding · Visual suffix $\cdot$ Filtering

We consciously see a world of complex objects, each comprising simple features such as color, shape, movement, texture, and depth. Thus, our brain has not only to represent these single features, but also to combine them appropriately into a unified pattern. However, each feature is assumed to be represented and processed separately in the brain (e.g., Desimone, Schein, Moran, \& Ungerleider, 1985), offering a problem of how the features that belong to a specific object are bound together while, at the same time, avoiding falsely binding features from different objects. This issue of binding is not confined to perception and has recently attracted considerable attention in the context of visual working memory, where the question is how we maintain the feature bindings of objects when they are out of sight for a short time. The aim of the present study was to address this broad question - in particular, exploring the nature of visual representations in working memory and the extent to which they are able to withstand interference from further stimuli.

Luck and Vogel (1997); Vogel, Woodman, and Luck (2001) argued that visual information is retained as a limited set of bound object representations. In a series of experiments using the change detection paradigm (Phillips, 1974; Phillips \& Baddeley, 1971), Luck and Vogel required participants to remember the contents of an initial stimulus array and then compare them with the contents of a second array, indicating whether they were the same or different. Crucially, they found that memory accuracy for presented 
features (e.g., color, orientation) did not vary when distinct objects each contained one to four features from different dimensions (color, size, orientation, presence/absence of a gap).

Although these findings were interpreted as evidence for an object-based visual store, it is also possible that performance in the Luck and Vogel (1997) experiments might reflect a set of distinct stores holding features such as color and shape separately, rather than bound object representations. In line with this, Treisman (e.g., Treisman 1988, 1998, 2006; Treisman \& Gelade, 1980) has developed a multilevel model of visual perception and short-term memory in which information is stored both in separate parallel feature maps and as bound object tokens. Treisman (1998) argued that both of these forms of representation can support memory for individual features and that such judgments can be made without necessarily requiring access to object representations. However, knowledge of how features are combined is critically dependent on the availability of these bound object representations. The evidence for object-level representations provided by Luck and Vogel, coupled with an apparent cost when objects consist of two features from the same dimension (e.g., two colors; Olson \& Jiang, 2002; Wheeler \& Treisman, 2002), would seem to support such a feature- and object-level approach (Jiang, Makovski, \& Shim, 2009).

It is therefore important to explore how representations at the bound object level may operate and interact with feature stores. There is already considerable evidence indicating that visual working memory is generally prone to information loss through retrograde interference (e.g., Broadbent \& Broadbent, 1981; Brockmole, Wang, \& Irwin, 2002; Jiang \& Kumar, 2004; Makovski, Sussman, \& Jiang, 2008). Furthermore, it is emerging that bound object representations are particularly fragile and susceptible to interference caused by the processing of subsequently encountered stimuli (Allen, Baddeley, \& Hitch, 2006; Logie, Brockmole, \& Vandenbroucke, 2009; Wheeler \& Treisman, 2002). For example, Wheeler and Treisman (2002) extended and modified the Luck and Vogel (1997) procedure, testing memory both for single features (color or shape) and for feature bindings (color and shape together). They also varied the size of the test array. Thus, in one condition, the test array included the same number of objects as the studied array, whereas in another, the test was a single probe item. Wheeler and Treisman found that changes in binding were less easy to detect than changes in single features when the test array included the same number of items as the studied array, but not when the test was a single probe item. This would indicate that the processing of multiple items at test disrupted the stored representations of bound objects, more so than individual features.
Further evidence for the fragility of bound representations was provided by Allen et al. (2006). They suggested that binding information is particularly susceptible to interference and overwriting caused by subsequent stimuli. In the key experiment, they compared memory for single features (color or shape) with memory for feature conjunctions (shape-color) when stimuli were presented either simultaneously or sequentially. The assumption was that study items presented early in a sequence would suffer from the presentation of later study items, whereas in the standard simultaneous presentation condition, such retroactive interference should be absent. If binding is indeed particularly fragile, it should be selectively reduced with sequential presentation, even when tested with a single probe. This proved to be the case. In addition, a clear serial position curve was obtained for hit rates, with lower accuracy in the binding condition in the earlier serial positions, consistent with the argument that the sequential decrement on binding memory performance was caused by retroactive interference from the later presented study items.

The aim of the present experiments was to explore the fragility of bound representations further, using a different methodology, and to aid the development of more detailed and constrained models of visual memory. Specifically, we examined the boundary conditions of this retroactive interference effect on memory for feature bindings, using the stimulus suffix paradigm. The stimulus suffix is a redundant item presented immediately after presentation of the to-be-remembered items. Although participants are instructed to ignore the suffix, it may nevertheless lead to systematic interference effects. Such effects are well established in the domain of auditory-verbal short-term memory (Crowder \& Morton, 1969). There is also a certain amount of evidence for visual suffix effects in memory for visually presented verbal items (Hitch, 1975; Kahneman, 1973) and for visuospatial information (Parmentier, Tremblay, \& Jones, 2004; Tremblay, Nicholls, Parmentier, $\&$ Jones, 2005). However, so far as we are aware, the visual suffix paradigm has not been used to investigate memory for visual features and feature bindings.

In our case, stimuli were presented simultaneously on screen, followed by a single visual suffix between the offset of these study items and the onset of a probe item testing recognition memory. On the basis of the findings of Wheeler and Treisman (2002) and Allen et al. (2006), it is suggested that the object/binding level is particularly fragile and vulnerable to interference and, so, will suffer more disruption than will memory for individual features when a suffix is presented during the short retention interval. In making such a prediction, we accept a multilevel structure of visual memory similar to that described by Treisman (Treisman 1988, 1998, 2006; Treisman \& Gelade, 1980) in feature integration theory. The assumption is that feature 
information (shape, color) is held in separate stores and as part of bound object representations, with memory for how features were specifically combined also represented in the latter form. Thus, information about features is represented at both feature and object levels, whereas binding information is represented only at the object level (Treisman, 2006). The lack of redundancy in the storage of binding information provides a possible explanation for its greater fragility and sensitivity to disruption from subsequent visual stimuli.

The suffix paradigm used in the present experiments also allows the separation of two possible mechanisms that might underlie the selective retroactive interference effect observed by Allen et al. (2006). In that study, the interfering items in the sequential presentation condition were also study items. Because these items needed to be remembered, it is not clear whether the retroactive interference observed was a result of intentional processing or reflected a relatively automatic influence of study items presented later in the sequence. In contrast, the interfering item in the suffix paradigm is to be ignored, and therefore, participants do not need to deliberately encode or store it in memory. Thus, any interference will be a consequence of encoding the suffix automatically or attempting to exclude it from entering memory.

Furthermore, by manipulating the nature of the suffix that is encountered, it is possible to explore the structure and function of the visual memory system and, in particular, the processes that determine how to-be-ignored stimuli are encoded into or excluded from visual memory. In Experiment 1A and 1B, we used the same suffix on each trial, with no overlap between the features of the suffix and the study items, In Experiment 2, we increased the variability of suffixes so as to be closer to the variability of the study items in the Allen et al. (2006) study, but with the features of suffixes again having no overlap with the set from which the study items were drawn. Finally, in Experiments $3 \mathrm{~A}$ and $3 \mathrm{~B}$, we constructed suffixes from the same set of features as that used for study items (although not allowing suffixes to contain features presented within the same trial). In each case, we compared the effects of these suffixes on feature and binding memory.

\section{Experiment 1A: consistent suffix with no feature overlap}

Method

\section{Participants}

Forty-two Kyoto University students $(17$ men and 25 women), between 18 and 26 years of age $(M=21.26$,
$S D=2.23$ ), were tested individually and were paid for attendance. All participants in this and the following experiments had normal color vision, as assessed by the Ishihara (1966) color blindness test.

\section{Materials}

Testing was controlled on a Windows PC with a 15-in. screen, using an HSP3 (hot soup processor, Version 3) program. All stimuli, probe items, and suffixes were simple colored shapes subtending a visual angle of approximately $0.75^{\circ}$, presented on a white background. The pool of study items was identical to that in Allen et al. (2006). Thus, 64 objects were formed by crossing eight colors (black, gray, blue, red, yellow, green, turquoise, and violet) and eight shapes (circle, chevron, triangle, diamond, star, cross, arch, and flag) (see the Appendix for illustrations). These stimuli were used in the binding condition. In the shape condition, unfilled shapes from the same set, with two-point black outlines, were used. In the color condition, stimuli were chosen from the set of eight colors and were all square shapes. Participants were shown all the possible stimuli, including suffixes, in the instruction phase.

The suffix was always a brown hexagon in the binding condition, a brown square in the color condition, and a line drawing of a hexagon in the shape condition. Thus, in this first experiment, the suffix features did not overlap with those of the targets. The visual angle of suffixes was the same as that of the to-be-remembered items $\left(0.75^{\circ}\right)$.

\section{Design and procedure}

The experiment used a 2 (suffix, no suffix) $\times 3$ (stimulus: color, shape, and binding) repeated measures design. Stimulus conditions were blocked and comprised 12 practice trials plus 80 test trials. The order of the three stimulus blocks was counterbalanced across participants. A rest was provided halfway through each block. Trials were evenly divided in each stimulus condition between randomly distributed same and different probes and also between randomly distributed suffix and no-suffix trials.

We adopted the same single probe recognition paradigm as that in Allen et al. (2006) and Wheeler and Treisman (2002). Figure 1 illustrates the time course of each trial, which began with a warning cross, presented for $500 \mathrm{~ms}$ in the center of the screen. Following a blank screen $(250 \mathrm{~ms})$, the study array was presented for $2,000 \mathrm{~ms}$. This array consisted of four stimuli in the center of the screen, presented in a row subtending an angle of $7.50^{\circ}$ in total, with $1.50^{\circ}$ between the edges of adjacent items $\left(2.25^{\circ}\right.$ between the centers of adjacent items). The two stimuli in the middle spatial positions were located with their nearest edge 0.75 from the center of the screen. In the no-suffix 


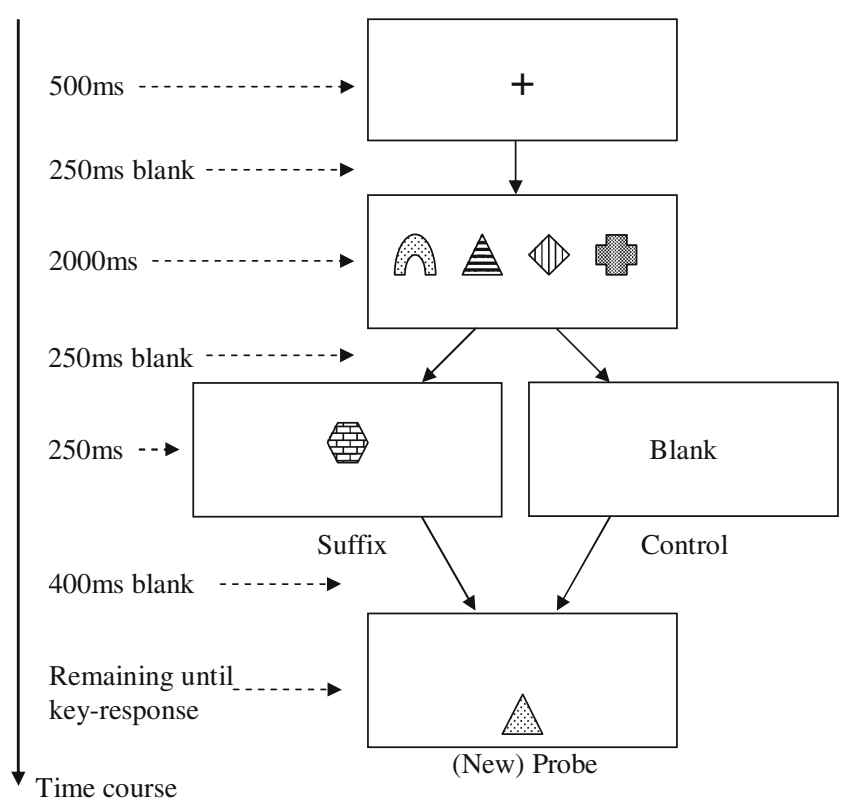

Fig. 1 Time course of a single trial in the binding condition of Experiment 1A. Different textures represent different colors

condition, a blank screen delay of $900 \mathrm{~ms}$ followed, before the test probe was presented just below center screen in a neutral, previously unoccupied location. In the suffix condition, a suffix was presented in the center of the screen during the 900-ms delay. The suffix was presented in the same row as the study stimuli, the visual angle between the center of the suffix and the center of the middle stimuli being $1.125^{\circ}$. The suffix was presented $250 \mathrm{~ms}$ after the offset of the study display and remained visible for $250 \mathrm{~ms}$. The timing of suffix presentation paralleled the interstimulus interval (ISI) in the sequential presentation condition of Allen et al. (2006), which was also $250 \mathrm{~ms}$. Participants were instructed to ignore a suffix when it was presented but not to close their eyes or to avert their gaze. In all conditions, the probe item remained visible until participants made their "same" or "different" response via a keypress. Accuracy was emphasized rather than speed. A warning cross signifying the next trial followed immediately.

Probes Same probes were randomly selected from the four stimuli in the study array, with the restriction that each location was selected with equal probability. Different probes in the color condition and shape conditions consisted of a color or a shape that had not been presented in the study phase. In the binding condition, different probes involved recombining a color and a shape that had appeared in different items in the study array.

Feature repetition Displays containing repetition of a feature (e.g,. two triangles) were included on $20 \%$ of the trials to ensure that correct rejection of different probes in the binding condition required memory for all the binding information in the display. Without the possibility of feature repetition, it may be possible to reject a lure probe (e.g., a red triangle) on the basis of memory for a single presented item containing one of the lure features (e.g, a. red circle; see Allen et al., 2006, for more details). Half of the practice trials involved feature repetition in order to discourage this strategy. The remaining $80 \%$ of the test trials involved no feature repetition. Only these trials were submitted to subsequent analysis.

Encoding strategy In order to discourage verbal coding, participants were required to repeat the sequence $1-2-3-4$ aloud at two digits a second from the warning cue until the test probe appeared. To promote articulatory suppression, a beep sounded twice with a 500-ms ISI at the onset and offset of the warning cross on each trial. A number of studies have shown that accuracy in visual short-term memory tasks is not significantly impaired by such articulatory suppression (Morey \& Cowan, 2004; Vogel et al., 2001).

\section{Results}

\section{Scoring}

For recognition accuracy, we calculated both a corrected recognition score, obtained by subtracting the proportion of false alarms from the proportion of hits, and $d$ ', based on signal detection theory. Analysis of the two scores did not differ, and thus we report only corrected recognition. For an effect size indicator, we report partial $\eta^{2}$ for analysis of variance (ANOVA) and Cohen's $d$ for $t$ tests (Cohen, 1988).

\section{Corrected recognition}

Figure 2 shows the corrected recognition rates in each stimulus condition. A 2 (suffix) $\times 3$ (stimulus condition) ANOVA indicated significant main effects of suffix, $F(1$, $41)=11.712, M S E=2.271 \mathrm{E}^{-2}, p=.001$, partial $\eta^{2}=.222$, and stimulus type, $F(2,82)=107.313, M S E=2.229 \mathrm{E}^{-2}$, $p<.001$, partial $\eta^{2}=.724$, and no significant stimulus $\times$ suffix interaction, $F(2,82)=2.260, M S E=1.964 \mathrm{E}^{-2}$, $p=.110$, partial $\eta^{2}=.052$. Presentation of a suffix impaired recall; memory for color was more accurate than memory for shape, and both were more accurate than memory for binding, all $t \mathrm{~s}(82)>6.686, M S E=0.228 \mathrm{E}^{-1}, p \mathrm{~s}<.001, d \mathrm{~s}=$ $1.204,1.986$, and 1.242, respectively (Ryan's multiple comparison procedure).

\section{Discussion}

We obtained a small but significant visual suffix effect indicating the usefulness of the suffix paradigm, consistent 


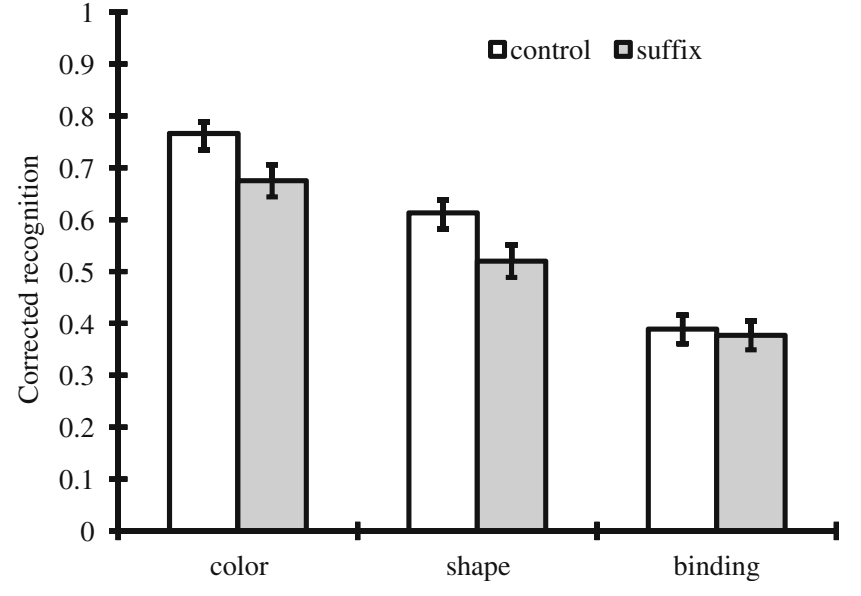

Fig. 2 Corrected recognition (hits - false alarms) and standard errors as a function of stimulus condition and suffix: Data from Experiment 1A

with the earlier literature on verbal and visual suffix effects (e.g., Crowder \& Morton, 1969; Hitch, 1975; Parmentier et al., 2004; Tremblay et al., 2005). Importantly, the absence of a significant interaction between the suffix factor and stimulus type indicates that a greater vulnerability of bound features to retroactive interference from stimuli presented later does not always occur. However, before exploring this further, it seemed advisable to ensure that our result would replicate. Although the interaction was not significant, there appears to be a trend toward larger suffix effects on feature memory than on binding memory. In Experiment 1B, we investigated whether this tendency was a reliable pattern, using an instruction emphasizing both speed and accuracy. This allowed us to report reaction times (RTs), as well as number of correct responses, which might potentially be more informative.

\section{Experiment 1B: replication under instructions emphasizing speed}

\section{Method}

\section{Participants}

Thirty-six Kyoto University students (19 men and 17 women) were tested individually and were paid for their attendance.

\section{Materials, design, and procedure}

The experiment used the same materials and design as before. The general procedure was identical to that in Experiment 1A, except that the instructions emphasized speed in addition to accuracy and there was a greater number of trials. Recognition RT was based on the time between the onset of a recognition probe and a keypress response and was measured with 1-ms sensitivity. Stimulus conditions were blocked and each contained 12 practice trials and 160 test trials.

Results

\section{Corrected recognition}

Figure 3 shows the corrected recognition rates in each stimulus condition. A two-way repeated measures ANOVA with stimulus and suffix as factors indicated significant main effects of both suffix, $F(1,35)=14.808, M S E=$ $1.022 \mathrm{E}^{-2}, p<.001$, partial $\eta^{2}=.297$, and stimulus type, $F(2,70)=149.017, M S E=1.541 \mathrm{E}^{-2}, p<.001$, partial $\eta^{2}=$ .809. As before, the presence of a suffix impaired recognition, whereas memory for color was more accurate than that for shape and both were more accurate than that for binding, all $t \mathrm{~s}(70)>4.090, M S E=1.541 \mathrm{E}^{-2}, p \mathrm{~s}<.001$, $d \mathrm{~s}=0.679,2.827$, and 2.040 , respectively. There was again no significant stimulus type $\times$ suffix interaction, $F(2,70)=$ $1.419, M S E=9.545 \mathrm{E}^{-3}, p=.248$, partial $\eta^{2}=.038$.

\section{Reaction time}

Figure 4 shows mean RTs for correct responses in each condition. A two-way repeated measures ANOVA revealed a significant main effect of stimulus type, $F(2,70)=$ 34.038, MSE $=5488.26, p<.001$, partial $\eta^{2}=.493$, but no significant main effect of suffix, $F=3.062, p>.088$, and no suffix $\times$ stimulus type interaction, $F=1.974, p=.146$. Ryan's multiple comparison procedure indicated that latencies to color were shorter than those to shape or binding, both $t \mathrm{~s}(70)>6.821, M S E=5488.26, p \mathrm{~s}<.001$,

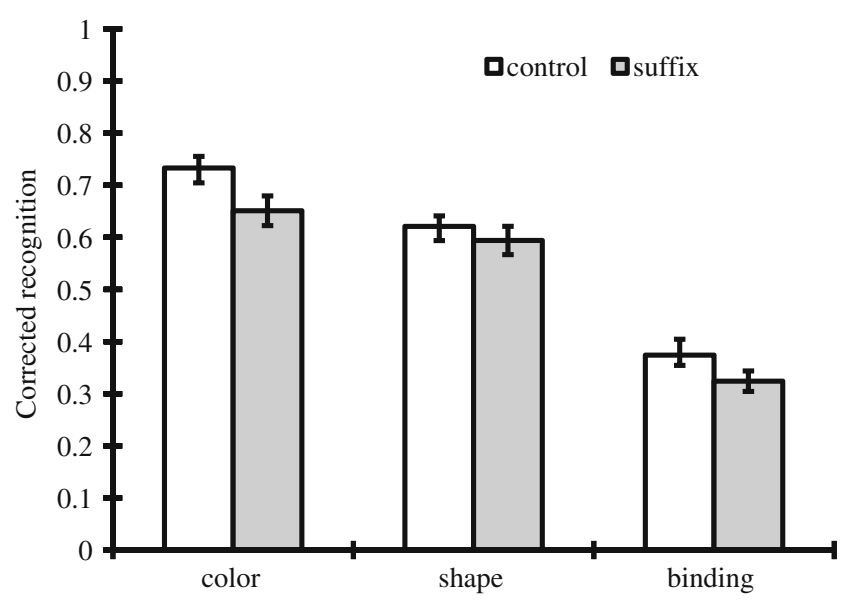

Fig. 3 Corrected recognition (hits - false alarms) and standard errors as a function of stimulus condition and suffix: Data from Experiment 1B 


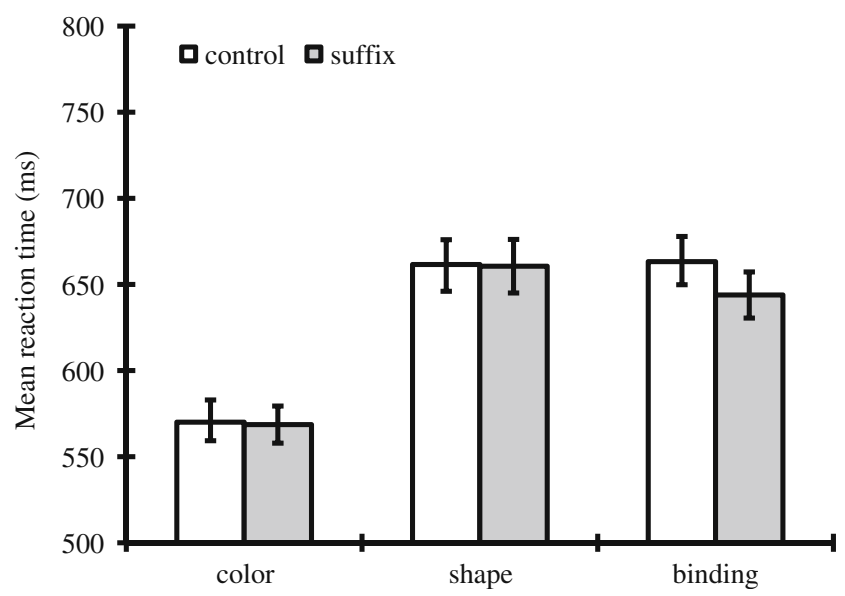

Fig. 4 Mean reaction times and standard errors as a function of stimulus condition: Data from Experiment 1B

$d \mathrm{~s}=1.197$ and 1.289 , respectively. Shape and binding latencies did not statistically differ, $t<1$.

\section{Discussion}

All of the main effects found in Experiment 1A were replicated, with a visual suffix effect on accuracy across all stimulus conditions and performance for color exceeding that for shape, which, in turn, was higher than that in the binding condition. We again failed to detect a significant interaction between suffix and stimulus type. Furthermore, the nonsignificant trend toward a smaller suffix effect on binding that was observable in Experiment $1 \mathrm{~A}$ was not seen in this experiment. Finally, RT data indicated that suffix presentation did not affect speed of responses across the three stimulus conditions.

Taking the results from Experiments $1 \mathrm{~A}$ and 1B together, we can reliably argue that the suffixes we used had an equivalent effect on feature memory and on memory for feature bindings. Thus, a greater vulnerability of bound features to retroactive interference from stimuli presented later does not always occur. There were, however, a number of methodological discrepancies between our suffixes and those in Allen et al.'s (2006) sequential presentation condition that might potentially have attenuated the suffix effect. One of these was the degree of suffix variability. This was the focus of Experiment 2.

\section{Experiment 2: variable suffixes with no feature overlap}

Since the interfering items in the sequential presentation conditions in Allen et al. (2006) were to-be-remembered targets, they necessarily involved new feature combinations on each trial. For example, an interfering study item might be a red circle, whereas on another trial, it might be a red triangle or a blue circle. Participants thus needed to form new bindings of these features on every trial, and this itself might contribute to the greater disruption in binding memory. In Experiments $1 \mathrm{~A}$ and 1B, however, there was no need to form new bindings for the suffix, since it was always the same item within each condition.

In Experiment 2, we introduced variation to suffixes, testing the effect of this on memory for shape, color, and shape-color bindings. To achieve this, suffixes were created by randomly sampling their shape and color from a pool of potential features. Note, however, that neither the colors nor the shapes of suffixes overlapped with the colors or shapes of study items.

If the selective retroactive interference effect found by Allen et al. (2006) with sequential presentation occurred because later items had variable combinations of features, a similar effect should be observed with variable suffixes. Thus, we were once again interested in whether presentation of a suffix would disrupt memory for feature bindings more than memory for individual features.

\section{Method}

\section{Participants}

Forty-two Kyoto University students (22 men and 20 women), between 18 and 28 years of age $(M=20.42$, $S D=2.44$ ), were tested individually and were paid for their attendance.

\section{Materials}

Study items were constructed in the same way as in Experiments 1A and 1B. Eighty-one suffixes were formed by crossing nine new colors and nine new shapes from outside the experimental pool. From this 81 -item pool, a subpool of nine suffixes was formed for each participant by crossing three randomly selected colors and three shapes (e.g., color 1 and shape 1 ; color 1 and shape $2 ; \ldots$; color 3 and shape 3 ). The visual angle subtended by suffixes was the same as that in Experiment 1. Examples are shown in the Appendix.

\section{Design and procedure}

As before, the experiment employed a 2 (suffix, no suffix) $\times 3$ (stimulus color, shape, and binding) repeated measures design. The procedure was also the same as that in Experiment 1A, apart from the change in type of suffix. Across three stimulus conditions, one of nine suffixes that made up each participant's suffix pool was randomly selected and presented on each trial. Thus, suffix variability operated across all three stimulus conditions. 
Results

\section{Corrected recognition}

Figure 5 shows the corrected recognition rates in each stimulus condition. A two-way repeated measures ANOVA with stimulus and suffix as factors indicated significant main effects of both suffix, $F(1,41)=13.309, M S E=$ $1.968 \mathrm{E}^{-2}, p<.001$, partial $\eta^{2}=.245$, and stimulus type, $F(2,82)=28.506, M S E=8.372 \mathrm{E}^{-2}, p<.001$, partial $\eta^{2}=$ .410. As before, the presence of a suffix impaired recognition. Memory was more accurate for color than for shape, and both were more accurate than that for binding, all $t \mathrm{~s}(82)>3.133, M S E=0.837 \mathrm{E}^{-1}, p \mathrm{~s}<.002, d \mathrm{~s}=0.464$, 1.062 , and 0.794 , respectively. There was again no significant stimulus type $\times$ suffix interaction, $F(2,82)=$ $0.125, M S E=2.466 \mathrm{E}^{-2}, p=.882$, partial $\eta^{2}=.003$.

\section{Discussion}

All of the main effects found in the previous experiments were replicated, with a visual suffix effect across all stimulus conditions and performance on color exceeding that on shape, which, in turn, was higher than that in the binding condition. We again failed to detect a significant interaction between suffix and stimulus type, suggesting that the presence of changing feature combinations in the interfering items was not sufficient to cause the differential disruption of binding found by Allen et al. (2006).

This result led us to consider another discrepancy between Allen et al. (2006) and our first three experiments - namely, the fact that the interfering items in the Allen et al. study were selected from the same pool as earlier items, whereas our suffixes were not. Hence, a variable suffix with features selected from the same pool as

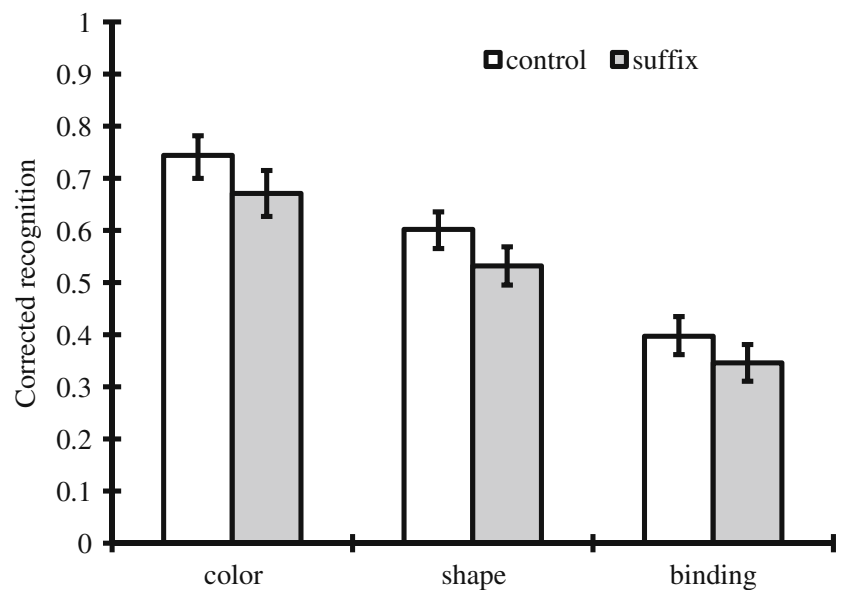

Fig. 5 Corrected recognition and standard errors as a function of stimulus condition and suffix: Data from Experiment 2 study items might have a selective interference effect on binding memory. We set out to test this hypothesis in the next experiment.

\section{Experiment 3A: variable suffixes with feature overlap}

In this experiment, the features of suffixes were selected from the same pool as study items. Thus, the suffix pool and the experimental pool overlapped completely. However, the features of the study items presented within a trial were never repeated in the following suffix. If the crucial aspect of the retroactive interference effect found by Allen et al. (2006) is that the later interfering items involved different combinations of the same set of features as those of earlier items, a corresponding effect should be observed when the suffix features were drawn from the same pool as study items. On the other hand, no such effect should be observed if the critical factor in Allen et al.'s (2006) result for sequential presentation was that later presented items had to be memorized intentionally.

\section{Method}

\section{Participants}

Forty-two students from the University of York (12 men and 30 women), between 18 and 38 years of age $(M=$ $20.07, S D=3.13$ ), were tested individually and were paid or given a course credit.

\section{Materials}

Study items were constructed in the same way as those in Experiments 1A, 1B, and 2. Suffixes were randomly selected on each trial from the experimental pool (64 objects for the binding condition, eight colors for the color condition, eight shapes for the shape condition), with the constraint that neither the color nor the shape of a suffix was present as a target feature within a trial.

\section{Factorial design and procedure}

The design and procedure were identical to those in Experiments $1 \mathrm{~A}$ and 2.

Results

\section{Corrected recognition}

Figure 6 shows corrected recognition rates in each stimulus condition. A two-way repeated measures ANOVA with stimulus type and suffix as factors revealed 


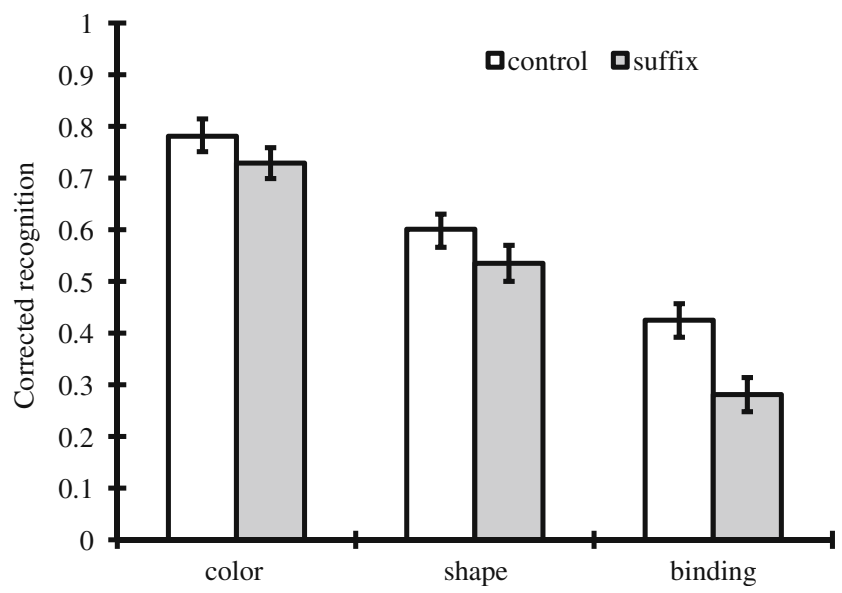

Fig. 6 Corrected recognition and standard errors as a function of stimulus condition and suffix: Data from Experiment 3A

significant main effects of suffix, $F(1,41)=28.301, M S E=$ $1.697 \mathrm{E}^{-2}, p<.001$, partial $\eta^{2}=.408$, and stimulus type, $F(2$, $82)=119.851, M S E=2.833 \mathrm{E}^{-2}, p<.001$, partial $\eta^{2}=.745$, and a significant two-way interaction, $F(2,82)=3.497$, $M S E=1.492 \mathrm{E}^{-2}, p=.035$, partial $\eta^{2}=.079$. Subsidiary analysis indicated that there was a significant simple main effect of suffix in the binding condition, $F(1,123)=28.040$, $M S E=1.560 \mathrm{E}^{-2}, p<.001$, partial $\eta^{2}=.185, d=0.835$, and in the shape condition, $F(1,123)=5.769, M S E=1.560 \mathrm{E}^{-2}$, $p=.017$, partial $\eta^{2}=.044, d=0.313$, and a marginally significant effect in the color condition, $F(1,123)=3.651$, $M S E=1.560 \mathrm{E}^{-2}, p=.058$, partial $\eta^{2}=.028, d=0.389$. Therefore, the significant interaction indicated that the effect size of the suffix factor was more marked in the binding condition. Finally, Ryan's multiple comparison procedure revealed that all pairwise differences between the three stimulus types were significant in both the suffix and no-suffix conditions, all ts $(164)>5.472, M S E=0.216 \mathrm{E}^{-1}$, $p \mathrm{~s}<.001, d \mathrm{~s}>0.931$.

\section{Discussion}

This experiment replicated the main effects of stimulus type and suffix obtained in the previous three experiments, confirming the robustness of the visual suffix effect across all stimulus conditions and once again showing the lowest accuracy for the binding condition. This time, however, by selecting a suffix from the same set as study items, we detected a clear increase in the amount of interference in the binding condition, which is parallel to the effect of sequential presentation observed by Allen et al. (2006). Despite the differences between the two studies, both show that the representations of feature bindings in visual working memory are more vulnerable to interference from subsequent items than are representations of individual features. In addition, the discrepancy between this result and those of Experiments 1A, 1B, and 2 clearly has implications for the nature of forgetting in visual working memory. However, before considering this question, it seemed advisable to ensure that our result would replicate. We attempted this in Experiment 3B, adding a further dimension by urging participants to respond rapidly as well as accurately, as we did in Experiment 1B.

\section{Experiment 3B: replication under instructions emphasizing speed}

The purpose of this experiment was to replicate the observation that a variable suffix drawn from the same pool as study items disrupts memory for feature bindings more than memory for individual features.

Method

\section{Participants}

Thirty-six students from Kyoto University (17 men and 19 women), between 18 and 25 years of age $(M=20.36, S D=$ $1.66)$, were tested individually and were paid for attendance.

\section{Factorial design and procedure}

The experiment used the same design as before. The general procedure was identical to that in Experiment $3 \mathrm{~A}$, with the exception of the instructions, which emphasized speed in addition to accuracy, and the number of trials (as was modified in Experiment 1B)

\section{Results}

\section{Corrected recognition}

Figure 7 shows the corrected recognition rates in each stimulus condition. A two-way (stimulus type $\times$ suffix) repeated measures ANOVA successfully replicated the findings from Experiment $3 \mathrm{~A}$. Thus, there were significant main effects of suffix, $F(1,35)=14.055, M S E=1.186 \mathrm{E}^{-2}, p<.001$, partial $\eta^{2}=.286$, and stimulus type, $F(2,70)=110.722$, $M S E=2.037 \mathrm{E}^{-2}, p<.001$, partial $\eta^{2}=.759$, and crucially, a significant interaction between suffix and stimulus type, $F(2$, $70)=4.595, M S E=7.194 \mathrm{E}^{-3}, p=.013$, partial $\eta^{2}=.116$. Subsidiary analyses indicated that there was a significant simple main effect of suffix in the binding condition, $F(1$, $105)=22.699, M S E=0.874 \mathrm{E}^{-2}, p<.001$, partial $\eta^{2}=.177$, 


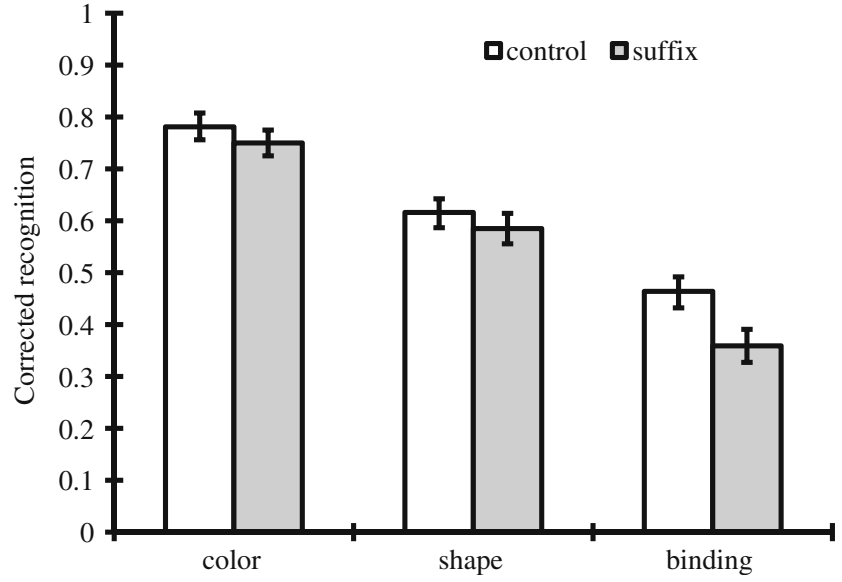

Fig. 7 Corrected recognition and standard errors as a function of stimulus condition: Data from Experiment 3B

$d=0.620$, but no effect in the shape condition, $F(1,105)=$ $1.899, M S E=0.874 \mathrm{E}^{-2}, p=.171$, partial $\eta^{2}=.017, d=0.253$, or in the color condition, $F(1,105)=2.009, M S E=0.874 \mathrm{E}^{-2}$, $p=.159$, partial $\eta^{2}=.017, d=0.383$. Thus, the significant interaction indicated that only binding memory suffered significantly from suffix interference. Finally, Ryan's multiple comparison procedure revealed that all pairwise differences between stimulus types were significant both in the with-suffix and in the no-suffix conditions, all $t \mathrm{~s}(140)>5.489, M S E=1.378 \mathrm{E}^{-2}, p \mathrm{~s}<.001, d \mathrm{~s}>1.022$.

\section{Reaction time}

Figure 8 shows mean RTs for correct responses in each condition. A two-way repeated measures ANOVA

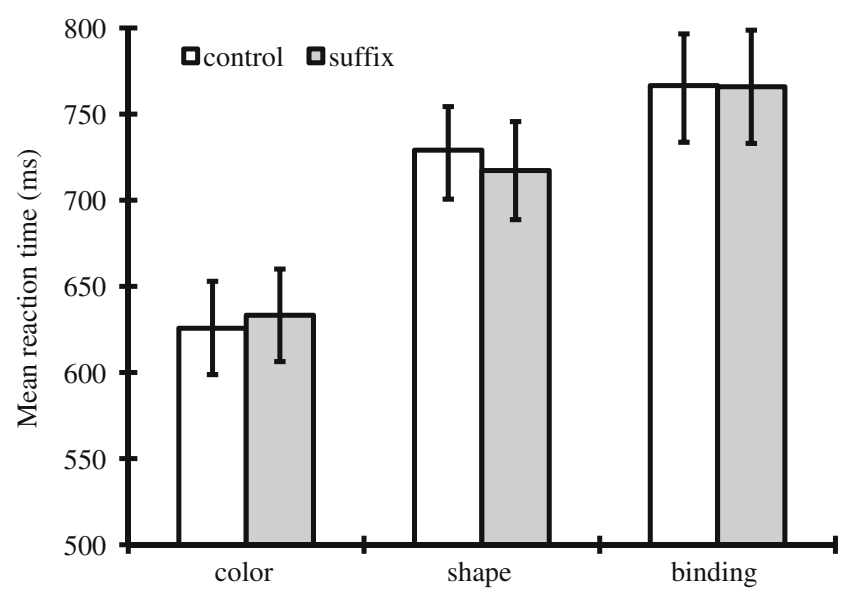

Fig. 8 Mean reaction times and standard errors as a function of stimulus condition: Data from Experiment 3B revealed a significant main effect of stimulus type, $F(2$, $70)=28.844, M S E=352073.93, p<.001$, partial $\eta^{2}=$ .452 , but no significant main effect of suffix and no suffix $\times$ stimulus type interaction, both $F_{\mathrm{S}}<1.197$. Ryan's multiple comparison procedure indicated that RTs for color were shorter than those for shape and that those for both color and shape were shorter than those for binding, all $t \mathrm{~s}(70)>2.338$, $M S E=12205.98, p \mathrm{~s}<.001, d \mathrm{~s}=0.984,1.136$, and 0.376, respectively.

\section{Cross-experiment comparison}

A cross-experiment analysis of the accuracy data from all five experiments revealed a significant three-way interaction across the factors of suffix, stimulus type, and experiment, $F(8,386)=2.217, M S E=1.561 \mathrm{E}^{-2}, p=.025$, partial $\eta^{2}=$ .043 , indicating reliable differential effects of suffixes from outside the experimental pool (Experiments 1A, 1B, and 2) and those from within the experimental pool (Experiments $3 \mathrm{~A}$ and $3 \mathrm{~B})$. The suffix effect significantly interacted with the experiment factor just in the binding condition, $F(4,579)=3.352, M S E=1.595 \mathrm{E}^{-2}, p=.010$, partial $\eta^{2}=$ .026 , whereas it did not significantly interact in the color and the shape conditions, both $F$ s $<1$. These patterns were replicated when we analyzed just the data from Experiments $1 \mathrm{~A}, 2$, and $3 \mathrm{~A}$, where only accuracy was emphasized.

\section{Discussion}

The results replicated the previous findings that a suffix containing features from the experimental pool interferes selectively with memory for feature bindings and established the robustness of this effect under instructions emphasizing speed as well as accuracy. The RT data indicated no corresponding interaction in speed of responding, rendering it unlikely that the interference effect in accuracy was due to a speed-error trade-off. Taken together with the crossexperiment analysis of the accuracy data, we conclude that there is a reliable difference in the effect of a suffix on memory for features and memory for feature bindings and that this difference is contingent on the features of the suffix being drawn from the same set as the study items. In contrast, a suffix containing features drawn from a different set from that for study items interferes with the accuracy of memory for features and feature binding to an equal extent.

\section{General discussion}

In an earlier study, Allen et al. (2006) observed that with sequential presentation, memory for feature bindings was more susceptible to disruption from later items than was memory for single features. We conducted five experiments 
to investigate this finding, using a paradigm in which an array of study items was followed by a single suffix item that was to be ignored. The characteristics of the suffix were varied between experiments. All five experiments obtained a significant effect of the suffix, although in the case of retention of individual features, this effect was small, regardless of the nature of the suffix. In the case of the bound features, however, a more complex pattern emerged. When the suffix comprised features that did not overlap with the study items, disruption was minimal and equivalent to that observed with individual features. However, when suffixes comprised features that could potentially be part of the remembered item set, degree of disruption increased, becoming significantly larger for feature bindings than for separate features. Our results thus replicate and extend those of Allen et al. (2006), demonstrating, first, that it is not necessary for the participant to attempt to remember an item for it to interfere with memory for previous items and, second, that the characteristics of the interfering item determine the extent to which memory for feature bindings is affected.

At the broadest level, we have established a limited but robust phenomenon that places constraints on any adequate theory of visual working memory. However, in the current state of knowledge, elaborating any such model would involve making numerous arbitrary assumptions. We therefore concentrate on identifying the general constraints our results place on theories of visual working memory before going on to consider in outline what kinds of mechanism might plausibly satisfy them. In terms of such constraints, our results argue for at least a two-factor account of visual suffix effects. One is a general factor responsible for the small but consistent suffix effects on memory for features and bindings observed across experiments. The other is a specific factor responsible for the change in the pattern of suffix effects, whereby memory for bindings was found to be especially vulnerable when suffixes contained features from the same pool as study items.

Any post hoc interpretation of the kinds of mechanisms that might have generated our results is inevitably somewhat speculative. In our preferred account, we assume that the general factor reflects a filtering process set up to exclude the suffix from entering visual working memory, whereas the specific factor corresponds to interference within memory when the filter fails. The concept of a filter is supported by neurophysiological evidence that the efficiency of keeping out irrelevant items is a primary source of individual differences in the storage capacity of visual working memory (Vogel, McCollough, \& Machizawa, 2005). We assume that the filtering process is visuospatial but under the control of general executive resources, depending, as it does, on the task instruction to ignore the suffix. Hence, there is a general attentional cost to setting up and running the filter, which results in a modest disruption of memory performance. Given that an encoding and retention-based concurrent executive task impairs memory for features and feature bindings to the same extent (Allen et al., 2006, Experiments 2-4), we expect the cost of running the filter to be the same for memory for features and bindings. If we make the plausible assumption that the filter can readily exclude a suffix with either a predictable appearance (as in Experiments 1A and 1B) or features that render the object implausible as a potential study item (as in Experiment 2), we can account for the small but equivalent suffix effects on memory for features and feature bindings under these conditions.

We account for the contrasting effects of plausible suffixes containing features from the same set as study items (as in Experiments 3A and 3B) in terms of a second factor corresponding to what happens when the filter fails. We assume that a plausible suffix will sometimes pass through the filter and gain access to memory, where it will tend to overwrite stored information. We assume further that there are different levels of representation in visual working memory corresponding to features and objects, with feature information represented at both levels and binding information only at the object level. Binding information is therefore stored with less redundancy than is feature information and so is more susceptible to overwriting. In this way, we can suggest a plausible account for the greater effect of a plausible suffix on memory for bindings than for individual features.

Our interpretation can be seen as consistent with the multilevel feature integration theory developed by Treisman (e.g., Treisman 1988, 1998, 2006; Treisman \& Gelade, 1980; see also Olson \& Jiang, 2002). The idea that binding information is particularly susceptible to overwriting is evidently fully consistent with the evidence of poorer memory for feature bindings than for individual features for items presented early in a sequence (Allen et al., 2006, Experiment 5). Less obviously, it is also consistent with the binding decrements with whole test displays observed by Wheeler and Treisman (2002). The latter can be viewed as reflecting a process of overwriting by multiple "suffixes," rather than the withdrawal of general attention (see also Treisman, 2006, for a similar interpretation).

To summarize, we suggest that the nonselective effect of a visual suffix on memory for features and bindings can be attributed to the executive cost of filtering out the suffix. On the other hand, the selective effect of a plausible suffix on memory for bindings can be attributed to the increased likelihood of such a suffix passing the filter and overwriting stored information, coupled with the greater sensitivity of binding information to interference. To evaluate the strength of this interpretation, it is instructive to consider an alternative 
class of explanations that makes converse assumptions about the general and specific factors. That is, one could assume that filtering is selective, affecting memory more for bindings than for features, whereas overwriting is nonselective, disrupting memory for features and bindings to an equal extent. For example, one could argue that filtering out plausible suffixes is more demanding of general attention than is filtering out implausible suffixes and, following Wheeler and Treisman (2002), that maintaining object-level representations requires more attention than does maintaining single features. Thus, the greater attentional demands of filtering out plausible suffixes would cause bound representations to be neglected and, consequently, to disintegrate. One only has to add to this account the assumption of a general form of retroactive interference due to inadvertent processing of the suffix to explain the constant effect of any suffix on any form of memory.

However, other evidence somewhat undermines the argument that the fragility of memory for bindings reflects a withdrawal of attentional resources. We have already noted our own work showing that binding memory was no more affected than memory for features when general attention was occupied by the requirement to perform a demanding concurrent task during encoding and retention of stimuli (Allen et al., 2006, Experiments 2-4; see also Delvenne, Cleeremans, \& Laloyaux, 2010, for evidence on the withdrawal of attention). Furthermore, the suggestion that the greater fragility of binding memory is due to increased attentional demands of filtering out similar suffixes predicts that the impairment in memory for binding will depend on the degree of similarity of the suffix to the study items. In recent unpublished experiments, Ueno, Mate, Allen, Hitch, and Baddeley (submitted) included an intermediate condition in which suffixes contained one plausible feature (from the same pool as study items) and one implausible feature (from a different pool from study items). The binding deficit was just as big when one feature of the suffix was plausible as when both features were plausible. This is inconsistent with a similarity-based account of the demands of filtering out a suffix, which would predict a smaller binding deficit, but is consistent with an account in which the presence of one or more plausible features is sufficient for a suffix to pass the filter and interfere with binding information. Thus, on balance, we favor the view that memory for bindings is distinguished by its sensitivity to overwriting by subsequent visual stimuli (as was proposed by Allen et al., 2006) and is not especially dependent on general attention (as was proposed by Wheeler \& Treisman, 2002).

However, regardless of whether the disruption of binding information reflects overwriting or the withdrawal of attention, the present experiments have been successful in applying the visual suffix paradigm to the question of how features and objects are represented in working memory. This technique has been useful in allowing us to distinguish between a general process that affects memory for features and objects to a similar extent and a more specific process that affects memory for objects more than memory for individual features. We can also conclude with some confidence that any satisfactory theoretical model of visual working memory will need to distinguish between and specify the effects of having to filter unwanted stimuli from entering memory and the consequences when filtering fails.

Acknowledgements We are grateful to the three anonymous reviewers for raising very useful and constructive comments. Also, we are extremely thankful to Kakeru Asaoka, Tomoko Matsunaga, and Humiyo Yoshikawa (Kyoto University) for their invaluable assistance in collecting data.

\section{Appendix}

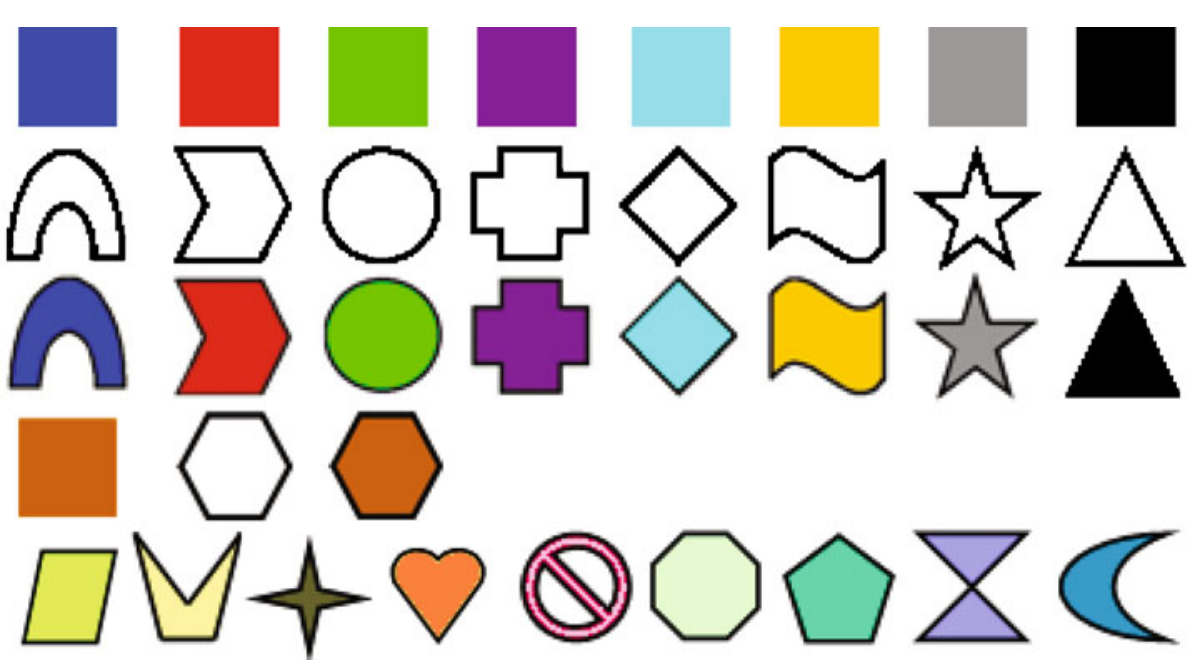


Top row: The eight colors used as targets in the color condition were, from the left, $\mathrm{RGB}=0,0,255$ for blue; 255, 0,0 for red; $0,255,0$ for green; 153, 0,153 for violet; $0,255,255$ for aqua; 255, 204, 0 for yellow; 153, 153, 153 for gray; and $0,0,0$ for black. Second row: The eight shapes used as targets in the shape condition. Third row: Examples of targets used in the binding condition. Fourth row: The three suffixes used in Experiments $1 \mathrm{~A}$ and $1 \mathrm{~B}$ ( $\mathrm{RGB}=204,102,0$ for brown). Bottom row: Examples of the nine unsaturated colors and the nine shapes used for suffixes in Experiment 2 (from the left, RGB > 204, 255, 102 for lime-colored rhombus; 255, 255, 153 for khaki crown; 102, 102, 51 for dark-olive four-point star; 255, 204, 102 for orange heart; $255,0,102$ for pink stopping mark; 204, 255, 204 for light-green octagon; 0, 204, 153 for emerald pentagon; 153, 153, 255 for amethyst sandglass; and $0,153,204$ for denim moon).

\section{References}

Allen, R. J., Baddeley, A. D., \& Hitch, G. J. (2006). Is the binding of visual features in working memory resource-demanding? Journal of Experimental Psychology: General, 135, 298-313. doi:10.1037/0096-3445.135.2.298.

Broadbent, D. E., \& Broadbent, M. H. P. (1981). Recency effects in visual memory. Quarterly Journal of Experimental Psychology: Human Experimental Psychology, 33(A), 1-15.

Brockmole, J. R., Wang, R. F., \& Irwin, D. E. (2002). Temporal integration between visual images and visual percepts. Journal of Experimental Psychology: Human Perception and Performance, 28(2), 315-334. doi:10.1037/0096-1523.28.2.315.

Cohen, J. (1988). Statistical power analysis for the behavioral sciences (2nd ed.). Hillsdale, NJ: Erlbaum.

Crowder, R., \& Morton, J. (1969). Precategorical acoustic storage (PAS). Perception \& Psychophysics, 5, 365-373.

Delvenne, J. F., Cleeremans, A., \& Laloyaux, C. (2010). Feature bindings are maintained in visual short-term memory without sustained focused attention. Experimental Psychology, 57, 108116. doi:10.1027/1618-3169/a000014.

Desimone, R., Schein, S. J., Moran, J., \& Ungerleider, L. G. (1985). Contour, color, and shape analysis beyond the striate cortex. Vision Research, 25, 441-452.

Hitch, G. J. (1975). The role of attention in visual and auditory suffix effects. Memory \& Cognition, 3, 501-505.

Ishihara, S. (1966). Tests for colour-blindness. Toyko, Japan: Kanehara Shuppan.

Jiang, Y. V., \& Kumar, A. (2004). Visual short-term memory for two sequential arrays: One integrated or two separate representations? Psychonomic Bulletin \& Review, 11(3), 495-500.

Jiang, Y. V., Makovski, T., \& Shim, W. M. (2009). Visual memory for features, conjunctions, objects, and locations. In J. R. Brockmole (Ed.), The visual world in memory (pp. 33-65). Hove, UK: Psychology Press.

Kahneman, D. (1973). Attention and effort. Englewood Cliffs, NJ: Prentice-Hall.

Logie, R. H., Brockmole, J. R., \& Vandenbroucke, A. R. E. (2009). Bound feature combinations in visual short-term memory are fragile but influence long-term learning. Visual Cognition, 17, 160-179. doi:10.1080/13506280802228411.

Luck, S. J., \& Vogel, E. K. (1997). The capacity of visual working memory for features and conjunctions. Nature, 390, 279-281. doi: $10.1038 / 36846$.

Makovski, T., Sussman, R., \& Jiang, Y. V. (2008). Orienting attention in visual working memory reduces interference from memory probes. Journal of Experimental Psychology. Learning, Memory, and Cognition, 34(2), 369-380. doi:10.1037/0278-7393.34.2.369.

Morey, C. C., \& Cowan, N. (2004). When visual and verbal memories compete: Evidence of cross-domain limits in working memory. Psychonomic Bulletin \& Review, 11, 296-301.

Olson, I., \& Jiang, Y. (2002). Is visual short-term memory object based? Rejection of the "strong object" hypothesis. Perception \& Psychophysics, 64, 1055-1067.

Parmentier, F. B. R., Tremblay, S., \& Jones, D. M. (2004). Exploring the suffix effect in serial visuospatial short-term memory. Psychonomic Bulletin \& Review, 11, 289-295.

Phillips, W. A. (1974). On the distinction between sensory storage and short-term visual memory. Perception \& Psychophysics, 16, 283290.

Phillips, W. A., \& Baddeley, A. D. (1971). Reaction time and shortterm visual memory. Psychonomic Science, 22, 73-74.

Treisman, A. (1988). Features and objects: The fourteenth Bartlett memorial lecture. The Quarterly Journal of Experimental Psychology, 40(A), 201-237.

Treisman, A. (1998). Feature binding, attention and object perception. Philosophical Transactions of the Royal Society of London, B, 353, 1295-1306.

Treisman, A. (2006). Object tokens, binding, and visual memory. In H. D. Zimmer, A. Mecklinger, \& U. Lindenberger (Eds.), Handbook of binding and memory: perspectives from cognitive neuroscience (pp. 315-388). Oxford: Oxford University Press.

Treisman, A., \& Gelade, G. (1980). A feature integration theory of attention. Cognitive Psychology, 12, 97-136.

Tremblay, S., Nicholls, A. P., Parmentier, F. B. R., \& Jones, D. M. (2005). Visual distraction and visuo-spatial memory: A sandwich effect. Memory, 13, 357-363. doi:10.1080/09658210344000422.

Ueno, T., Mate, J., Allen, R. J., Hitch, G. J., \& Baddeley, A. D. (submitted). Interference with binding in visual working memory: a filtering mechanism excludes distracters with task-irrelevant visual features.

Vogel, E. K., McCollough, A. W., \& Machizawa, M. G. (2005). Neural measures reveal individual differences in controlling access to working memory. Nature, $438,500-503$. doi:10.1038/ nature 04171 .

Vogel, E. K., Woodman, G. F., \& Luck, S. J. (2001). Storage of features, conjunctions, and objects in visual working memory. Journal of Experimental Psychology: Human Perception and Performance, 27, 92-114. doi:10.1037/0096-1523.27.1.92.

Wheeler, M. E., \& Treisman, A. M. (2002). Binding in short-term visual memory. Journal of Experimental Psychology: General, 131, 48-64. doi:10.1037/0096-3445.131.1.48.

This work was supported by an award to the first author of a Research Fellowship of the Japan Society for the Promotion of Science (JSPS) for Young Scientists and by a grant to the first author from the 21st Century COE program (D-10 to Kyoto University), MEXT, Japan. The last author is supported by a grant from the Ministry of Education, Culture, Sports, Science, and Technology in Japan, Grant-in-Aid for Scientific Research (19530655). 